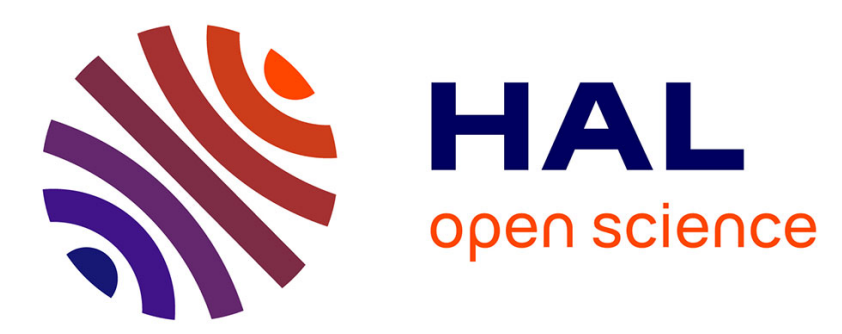

\title{
Computer-Aided 13 C NMR Chemical Profiling of Crude Natural Extracts without Fractionation
}

Ali Bakiri, Jane Hubert, Romain Reynaud, Sylvie Lanthony, Dominique Harakat, Jean-Hugues Renault, Jean-Marc Nuzillard

\section{To cite this version:}

Ali Bakiri, Jane Hubert, Romain Reynaud, Sylvie Lanthony, Dominique Harakat, et al.. ComputerAided 13 C NMR Chemical Profiling of Crude Natural Extracts without Fractionation. Journal of Natural Products, 2017, 80 (5), pp.1387 - 1396. 10.1021/acs.jnatprod.6b01063 . hal-01904954

\section{HAL Id: hal-01904954 \\ https://hal.univ-reims.fr/hal-01904954}

Submitted on 9 Sep 2021

HAL is a multi-disciplinary open access archive for the deposit and dissemination of scientific research documents, whether they are published or not. The documents may come from teaching and research institutions in France or abroad, or from public or private research centers.
L'archive ouverte pluridisciplinaire HAL, est destinée au dépôt et à la diffusion de documents scientifiques de niveau recherche, publiés ou non, émanant des établissements d'enseignement et de recherche français ou étrangers, des laboratoires publics ou privés. 
1 Computer-aided ${ }^{13} \mathrm{C}$ NMR chemical profiling of crude natural extracts without

2 fractionation

3

4 Ali Bakiri $^{\dagger, \S}$, Jane Hubert ${ }^{\dagger, *}$, Romain Reynaud ${ }^{\S}$, Sylvie Lanthony ${ }^{\dagger}$, Dominique Harakat ${ }^{\dagger}$, Jean-

$5 \quad$ Hugues Renault ${ }^{\dagger}$, Jean-Marc Nuzillard ${ }^{\dagger, \S}$

6

$7{ }^{\dagger}$ Institut de Chimie Moléculaire de Reims, UMR CNRS 7312, SFR CAP’SANTE, Université

8 de Reims Champagne-Ardenne, Reims, France

$9 \S$ Soliance-Givaudan, Pomacle, France 


\section{ABSTRACT}

2 A computer-aided, ${ }^{13} \mathrm{C}$ NMR-based dereplication method is presented for the chemical profiling

3 of natural extracts without any fractionation. An algorithm was developed in order to compare

4 the ${ }^{13} \mathrm{C}$ NMR chemical shifts obtained from a single routine spectrum with a set of predicted

5 NMR data stored in a natural metabolite database. The algorithm evaluates the quality of the

6 matching between experimental and predicted data by calculating a score function and returns

7 the list of metabolites which are the most likely to be present in the studied extract. The proof

8 of principle of the method is demonstrated on a crude alkaloid extract obtained from the leaves

9 of Peumus boldus, resulting in the identification of eight alkaloids including isocorydine,

10 rogersine, boldine, reticuline, coclaurine, laurotetanine, $N$-methylcoclaurine and

11 norisocorydine, as well as three monoterpenes including $p$-cimene, eucalyptol, and $\alpha$-terpinene.

12 The results were compared to those obtained with other methods, either involving a

13 fractionation step before the chemical profiling process or using mass spectrometry detection

14 in the infusion mode or coupled to gas chromatography.

15

16 KEYWORDS

17 Natural products, mixture analysis, dereplication, ${ }^{13} \mathrm{C}$ NMR, mass spectrometry, metabolite 18 database, Peumus boldus 


\section{1}

2 Natural product (NP) studies usually deal with samples of very complex chemical composition.

3 Isolation and structural elucidation of the metabolites within these mixtures remain tedious and

4 time consuming despite the advanced performance of modern chromatographic and analytical

5 technologies. One other major bottleneck of NP studies when searching for novel biologically

6 active substances and/or original chemical structures is the frequent rediscovery of very

7 common or already known metabolites, which causes a great waste of time. A good knowledge of the presence of known molecules within natural samples is therefore of great interest to speed up the chemical profiling of natural mixtures. This process of rapidly identifying known chemotypes, known as dereplication, has emerged over the last years as an essential approach to prevent duplication of isolation efforts. ${ }^{1-3}$

The most common detection techniques for the dereplication of NPs are mass spectroscopy (MS) and nuclear magnetic resonance (NMR), each one being very complementary to the other. ${ }^{1,2}$ Liquid or gas chromatography coupled to high-resolution MS are currently the most powerful "high-throughput screening" strategies for the on-line identification of metabolites in natural resources. ${ }^{4}$ Nevertheless, the important variability observed between MS datasets obtained from one mass analyzer to another and the tedious interpretation of MS data only based on elemental composition and fragmentation pattern are critical issues that limit the efficiency of MS-based déréplication. ${ }^{5}$ Efforts are currently underway to develop automatic processing methods, mainly through the implementation of computational treatments. Molecular networking in particular was recently validated as a promising computer-based approach to visualize and organize tandem mass spectrometry datasets and automate database search for metabolite identification within complex mixtures. ${ }^{6,7}$ Conversely, NMR is much less sensitive than MS, but remains, by far, the most efficient detection technique to unambiguously elucidate the molecular structures of secondary plant metabolite. ${ }^{8}$ A range of 1D and 2D NMR 
1 data acquisition sequences can be used to chemically profile natural samples via the 2 interpretation of ${ }^{1} \mathrm{H}-{ }^{1} \mathrm{H}$ and ${ }^{1} \mathrm{H}-{ }^{13} \mathrm{C}$ coupling patterns. ${ }^{9-13}$ Direct ${ }^{13} \mathrm{C}$ NMR detection provides a 3 large chemical shift dispersion $(240 \mathrm{ppm})$ that reduces signal overlaps and results in spectra 4 containing well-resolved individualized peaks due to broadband decoupling. The main 5 drawback of this technique is its low sensitivity due to a low natural abundance of the ${ }^{13} \mathrm{C}$ nuclei $6 \quad(\approx 1.1 \%)$ and its low magnetogyric ratio $\left(\approx 25 \%\right.$ of the one of the ${ }^{1} \mathrm{H}$ nucleus $)$. The detection 7 of quaternary carbon resonances which gives access to the whole carbon skeleton of metabolites 8 is another strong advantage of ${ }^{13} \mathrm{C}$ NMR, even though they cannot benefit from sensitivity 9 enhancement by coherent or incoherent magnetization transfer from ${ }^{1} \mathrm{H}$ nuclei. ${ }^{14}$ The relevance of ${ }^{13} \mathrm{C}$ NMR for mixture analysis has already been suggested in previous studies. For instance, 11 a very interesting algorithm was proposed in 1986 for the identification and quantification of organic mixture components using a quantitative ${ }^{13} \mathrm{C}$ NMR spectrum as input data. ${ }^{15}$ Unfortunately the method was tested on a model mixture of standard compounds and has never 14 been applied for the analysis of genuine mixtures of natural products. Another procedure based on ${ }^{13} \mathrm{C}$ NMR and computer tools was developed in 1994 to identify the most abundant phenolic derivatives in liquids produced by pyrolysis of biomass. ${ }^{16}$ The same method was applied to analyze the major chemical constituents of essential oils and was suggested as a useful complementary tool to GC/MS for the correct identification of isomers. ${ }^{17}$ In another work, highquality ${ }^{13} \mathrm{C}$ NMR data obtained with a ${ }^{13} \mathrm{C}$-optimized NMR probe were used to establish ${ }^{13} \mathrm{C}$ -

${ }^{13} \mathrm{C}$ statistical correlations in order to enhance the accuracy of metabolite identification at 21 natural abundance. ${ }^{14}$ Two years ago our laboratory developed a dereplication method based on 22 the combination of ${ }^{13} \mathrm{C}$ NMR with support-free liquid-liquid chromatography and Hierarchical 23 Clustering Analysis for the fast identification of the major metabolites in natural extracts. ${ }^{18}$ This 24 approach has since been successfully applied to several extracts containing different chemical 25 classes of secondary metabolites. ${ }^{19-22}$ With the same goal of maximizing the efficiency of NP 
1 dereplication, this paper presents a computer-aided ${ }^{13} \mathrm{C}$ NMR dereplication workflow that does

2 not require any pre-fractionation of the investigated sample in order to rapidly identify the major

3 constituents of a natural mixture while reducing as much as possible isolation steps. The proof

4 of principle of this algorithm is demonstrated on a crude alkaloid extract obtained from the

5 leaves of Peumus boldus (Monimiaceae), a small tree native to the central region of Chile that

6 is traditionally used for its digestive and hepato-biliary protective effects. ${ }^{23}$

7

\section{RESULTS AND DISCUSSION}

As the ${ }^{13} \mathrm{C}$ NMR fingerprint of a metabolite corresponds to an invariant set of chemical shifts that corresponds to its carbon skeleton, the ${ }^{13} \mathrm{C}$ NMR signals that originate from a single compound can be assigned to the corresponding metabolite even if this metabolite is mixed with other constituents, provided of course that there is a way to aggregate these signals. In this context an algorithm was developed in order to identify the major constituents of a crude natural mixture from a single ${ }^{13} \mathrm{C}$ NMR analysis on the basis of chemical shifts comparison between experimental and predicted datasets. This dereplication process involving the calculation of score values to evaluate each match between database records and the crude extract spectrum was tested on a crude alkaloid extract obtained from Peumus boldus leaves.

For data acquisition, ${ }^{13} \mathrm{C}$ NMR signals were recorded with broadband proton decoupling mode, in order to obtain a simplified ${ }^{13} \mathrm{C}$ NMR spectrum where each carbon atom of the metabolite mixture corresponds to a single sharp peak with the greatest possible intensity even for the quaternary resonances. Multiplicity edition by the J-modulated spin echo method (J-MOD) or the Distortionless Enhancement by Polarisation Transfer method (DEPT) would have indicated the parity of the number of protons attached to each carbon atom, and helped confirming or excluding proposals from database at the end of the dereplication process. J-MOD and DEPT pulse sequence rely on a spin echo module tuned for an average coupling constant ${ }^{1} J\left({ }^{1} \mathrm{H}-{ }^{13} \mathrm{C}\right)$ 
1 value and are therefore less sensitive than the simple ${ }^{1} \mathrm{H}$-decoupled ${ }^{13} \mathrm{C}$ sequence that was

2 retained for this study

3 The ${ }^{13} \mathrm{C}$ NMR spectrum of the crude Peumus boldus extract is presented in Figure 1. The

4 automatically produced peak list was directly used as an input file submitted to the algorithm.

5 The principle was to perform a search over a spectral database containing the structures and

6 predicted chemical shifts of all metabolites already reported in the genus Peumus $(\mathrm{n}=58)$ and

7 to compare the ${ }^{13} \mathrm{C}$ NMR chemical shifts of the crude extract spectrum to the predicted chemical

8 shifts of the database records. For practical reasons, we used for this purpose the NMR

9 Workbook Suite commercial software from ACD/Labs comprising in a single toolbox a

10 chemical structure drawing interface, a ${ }^{1} \mathrm{H} /{ }^{13} \mathrm{C}$ NMR prediction software and a spectral

11 database. The ACD/Labs prediction software is based on both the Hierarchical Organisation of

12 Spherical Environments (HOSE) $\operatorname{code}^{24}$ and neural network algorithms and takes advantage of

13 a considerable amount of literature data (more than 200,000 chemical structures for ${ }^{13} \mathrm{C}$ NMR

14 prediction with more than 2,000,000 ${ }^{13} \mathrm{C}$ NMR chemical shifts). To our three year experience,

15 the predicted values obtained with this software are quite reliable and the user interfaces are

16 easy to manage routinely. However, this software remains primarily a commercial product with

17 very limited access to the calculation details to obtain the predicted values. A few other efficient

18 and open source prediction tools would probably have been of great value and possibly

19 implemented in the proposed dereplication workflow, provided that some parts of the database

20 search algorithm be adapted accordingly. One can mention the NMRShiftDB web database ${ }^{25}$

21 which also involves the use of increment methods for NMR chemical shift prediction, or several

22 quantum chemical methods based for instance on the ab initio Hartree-Fock theory, second-

23 order Moller-Plesset theory or Density Functional theories (DFTs) which have been recognized

24 to yield good matching between experimental and computed values. ${ }^{25-27}$ 
1 With the database search algorithm proposed in the present work and applied to the ${ }^{13} \mathrm{C}$ NMR

2 peak list of the crude extract spectrum of Peumus boldus leaves, a total of 33 metabolites were

3 proposed with score values higher than 0.9 (Table 1). As the score value is defined as the ratio

4 between the numbers of chemical shifts of the crude extract that match a molecular structure of

5 the database and the total number of carbon positions of this structure, a score value higher than

60.9 means that more than $90 \%$ of the carbon positions of the metabolites proposed by the

7 database matched the experimental NMR signals of the crude extract spectrum. Among these

8 metabolites, a range of alkaloids including isocorydine (1), rogersine (2), boldine (3),

9 reticuline (4), coclaurine (5), laurotetanine (6), norisocorydine (7), and N-methylcoclaurine (8)

10 were found with a score value of 1 , indicating a complete matching of their predicted ${ }^{13} \mathrm{C}$ NMR

11 chemical shifts with the ${ }^{13} \mathrm{C}$ NMR signals detected in the crude extract spectrum. An alkaloid,

12 pronuciferine, showed a score value of 0.94 with only one mismatching chemical shift value

13 (Table 1). A mismatch may arise from important chemical shift differences between predicted

14 and experimental values or to non-homogenous peak intensity values resulting for instance from

15 quaternary or symmetrical carbon positions, therefore all chemical structures displaying score

16 values ranging from 0.9 and 1 were examined to confirm or not the presence of the proposed

17 metabolites. This step was performed by manually checking the ${ }^{13} \mathrm{C}$ NMR chemical shifts of

18 the matching chemical structures in the crude extract spectrum. The major alkaloids $\mathbf{1 - 8}$ were

19 easily confirmed. Pronuciferine was rapidly eliminated from the list of proposals because this

20 alkaloid contains a carbonyl group for which the characteristic resonance around 185 ppm was

21 not found in the crude extract spectrum. For such metabolites displaying score values comprised

22 between 0.9 and 1 , further refinement of the approach including for instance Density Functional

23 Theory (DFT) calculations would be useful to minimize bias and improve the reliability of the

24 prediction of NMR chemical shifts. ${ }^{25}$ Sans opinion... Rev2 sera content... 
1 In order to compare the results obtained by the ${ }^{13} \mathrm{C}$ NMR-based database search algorithm with

2 another method, the crude extract was solubilized in methanol and directly infused in a QToF

3 mass spectrometer. The resulting MS spectrum is presented in Figure 2. Several intense even

4 molecular ions corresponding to nitrogen-containing compounds were detected. The ion at $\mathrm{m} / \mathrm{z}$

5330.1 was attributed to the parent ion $[\mathrm{M}+\mathrm{H}]^{+}$of reticuline (4) and the ion at $\mathrm{m} / \mathrm{z} 286.1$ was

6 attributed to the parent ion $[\mathrm{M}+\mathrm{H}]^{+}$of coclaurine (5), thus confirming their presence in the crude

7 extract as revealed by the database search algorithm. Another intense ion detected at $\mathrm{m} / z 328.1$

8 could be attributed to the parent ion $[\mathrm{M}+\mathrm{H}]^{+}$of norisocorydine, boldine or laurotetanine, all

9 sharing the same molecular formula $\mathrm{C}_{19} \mathrm{H}_{21} \mathrm{NO}_{4}$. Unfortunately, it was impossible to

10 discriminate between these three isomers by this method. Similarly, it was impossible to

11 distinguish between isocorydine and rogersine for the attribution of the molecular ion detected

12 at $m / z, 342.1$ because these two alkaloids share the $\mathrm{C}_{20} \mathrm{H}_{23} \mathrm{NO}_{4}$ molecular formula. Yet mass

13 spectrometry was here a good method to rapidly and sensitively detect a pool of alkaloids in

14 the crude extract of Peumus boldus leaves. But, in total, only two alkaloids of the eight proposed

15 by the ${ }^{13} \mathrm{C}$ NMR-based database search algorithm were confirmed. However, without more

16 detailed structural data, it remained very difficult to discriminate between the different other

17 molecular structures, and even hyphenated to liquid chromatography, standards molecules

18 would have been necessary to unambiguously identify the individual alkaloids present in the

19 extract. The database search algorithm described in this work thus appears as a highly useful

20 complementary tool to MS for the chemical profiling of natural extracts.

21 In order to go further, another ${ }^{13} \mathrm{C}$ NMR-based dereplication method including a fractionation

22 step was applied on the same extract. For this purpose, a $\mathrm{pH}-z o n e$ refining Centrifugal Partition

23 Chromatography (CPC) (REF J.-H. Renault, G. Le Crouerour, P. Thépenier, J.-M. Nuzillard,

24 M. Zèches-Hanrot, L. Le Men-Olivier, Isolation of indole alkaloids from Catharanthus roseus

25 by centrifugal partition chromatography in the $\mathrm{pH}$-zone refining mode, J. Chromatogr. A 849 
1 (1999) 421-431.) method was developed to fractionate Peumus boldus alkaloids on the basis of

2 their proton affinity and distribution coefficient. It may be mentioned here that it is the first

3 time that Peumus boldus alkaloids were separated by CPC. The experiment was performed

4 using a biphasic solvent system primarily composed of $\mathrm{M} t \mathrm{BE}$ and water while $\mathrm{CH}_{3} \mathrm{CN}$ was

5 added as bridge solvent to reduce the polarity difference between the stationary and mobile

6 phases and ensure the solubility of all substances. The aqueous stationary phase was

7 supplemented with methane sulfonic acid (MSA) in order to ensure the protonation of all

8 alkaloids and therefore to promote their trapping into the aqueous stationary phase.

9 Triethylamine (TEA) was added to the organic mobile phase in order to progressively neutralize

10 the retainer and to selectively displace the alkaloids from the stationary to the mobile phase

11 according to their partition coefficients and acidity constants. As a result, the column effluent

12 remained at $\mathrm{pH} 2$ for 45 minutes, then increased to $\mathrm{pH} 6$ during the displacement of alkaloids

13 from 45 to 90 minutes, and finally increased up to $\mathrm{pH} 10$ when the $\mathrm{CPC}$ fractionation was

14 completed. Eleven fraction pools $\mathrm{P}_{\mathrm{I}}-\mathrm{P}_{\mathrm{XI}}$ were obtained and analyzed by ${ }^{13} \mathrm{C} \mathrm{NMR}$. Automatic

15 peak picking and alignment of ${ }^{13} \mathrm{C}$ NMR signals across spectra of the fraction series resulted in

16 a table with 11 columns (one per fraction) and 193 rows (one per chemical shift bin containing

17 at least one ${ }^{13} \mathrm{C}$ NMR signal in at least one fraction). The table was submitted to Hierarchical

18 Clustering Analysis (HCA) on the rows. As a result, statistical correlations between ${ }^{13} \mathrm{C}$ NMR

19 resonances belonging to a single structure within the fraction series were visualized as

20 "chemical shift clusters" in front of the corresponding dendrograms (Figure 3). Cluster A

21 corresponded to an intense cluster of $18{ }^{13} \mathrm{C}$ NMR chemical shifts. After entering these chemical

22 shifts into the database, the structure of isocorydine (1) was proposed as the first hit of over 21

23 proposals. This structure was confirmed by checking all chemical shifts of isocorydine in raw

24 NMR data of $P_{I}$ where the intensity of cluster 1 was predominant. Using the same method,

25 clusters $\mathrm{B}+\mathrm{B}^{\prime}$ were identified as a mixture of $N$-methylcoclaurine $(\mathbf{8})$ and reticuline (4). These 
1 two metabolites have very similar molecular structures and were detected collected? in the same

2 fraction pools, thus their ${ }^{13} \mathrm{C}$ NMR chemical shifts were aggregated under the same clusters.

3 The other clusters highlighted on the HCA correlation map were identified as coclaurine (5),

4 rogersine (2), norisocorydine (7), laurotetanine (6), and boldine (3), as indicated in Figure 3.

5 The molecular structures of all these compounds were confirmed by checking NMR data of the

6 corresponding fractions. Yet, the eight alkaloids identified by the database search algorithm

7 directly from the ${ }^{13} \mathrm{C}$ NMR analysis of the crude Peumus boldus extract without separation were

8 also identified by the ${ }^{13} \mathrm{C}$ NMR-based dereplication method involving a fractionation step. On

9 the one hand, this means that time consuming separation procedures are not always necessary

10 to identify the major constituents of a crude mixture. Applying the database search algorithm

11 just after a single ${ }^{13} \mathrm{C}$ NMR analysis of an extract can help to initiate a good chemical profiling

12 process while saving time, materials and solvents and giving useful information for further

13 investigations such as biological or toxicological evaluation, fractionation and purification

14 process development. On the other hand, it must be noted that the signal-to-noise $(\mathrm{S} / \mathrm{N})$ ratios

15 of all ${ }^{13} \mathrm{C}$ NMR spectra recorded after CPC fractionation were much higher than that of the

16 crude extract spectrum (Figure 5This brings us to the question of the minor constituents that

17 would be difficult to unambiguously identify by applying the database search algorithm just

18 after a single ${ }^{13} \mathrm{C}$ NMR analysis. Nevertheless, there is nothing to prevent the use of such

19 database search algorithm on fractions of simplified composition enriched with the minor

20 constituents of the extract. Mais Caramel ne permet pas de trouver d'autres constituants

21 minoritaires dans ce cas...

22 In addition to the eight alkaloids reported above, 24 monoterpenes were also proposed by the

23 database search algorithm, all with a score value of 1 , excepted for $\alpha$-copaene and $\beta$ -

24 oploplenone, $\delta$-cadinene and bornyl acetate which were effectively revealed absent from the

25 Peumus boldus extract after checking their chemical shifts in the experimental ${ }^{13} \mathrm{C}$ NMR 
1 spectrum. As carried out for alkaloids, molecular structure validation of the 20 monoterpenes

2 showing a score value of 1 was tentatively performed with the NMR dataset obtained after CPC

3 fractionation of the Peumus boldus extract. Unfortunately, only NMR signals of very low

4 intensity that could potentially correspond to monoterpenes were detected in the spectra of the

5 CPC fractions, thus making their interpretation impossible. The very low intensity of

6 monoterpene signals in the NMR spectra of the CPC fractions was probably due to the volatility

7 of these compounds, which resulted in their loss during fractionation and under-vacuum mobile

8 phase elimination. As an alternative, the crude Peumus boldus extract was analyzed by GC/MS.

9 As illustrated in Figure 4, several well-resolved monoterpene signals were obtained on the

resulting chromatogram. The retention times, molecular formula and electron ionization spectra

11 of these GC/MS-detected monoterpenes are given in Table 2. By comparing the MS spectra of

12 the different peaks of the chromatogram to the NIST MS Search library, six monoterpenes were

13 putatively identified among which $p$-cimene (9), eucalyptol (10), and $\alpha$-terpinene (11) were

14 common to the list of hits proposed by our database search algorithm. The identity of the other

monoterpenes proposed by the score function approach were not confirmed, either because the electron ionization MS spectra of the GC/MS-detected monoterpenes did not exactly match the spectra of the NIST MS Search library, or because the monoterpenes of the list of hits were absent from this library.

In summary, a database search algorithm was developed in order to chemically profile the major metabolites of a natural extract from a ${ }^{13} \mathrm{C}$ NMR analysis and without physical separation of the constituents. This algorithm was tested on a crude extract obtained from Peumus boldus leaves, leading to the successful identification of eight alkaloids including isocorydine (1), rogersine (2), boldine (3), reticuline (4), coclaurine (5), laurotetanine (6), norisocorydine (7), and $\mathrm{N}$-methylcoclaurine $(\mathbf{8})$ as well as a range of monoterpenes, among which para-cymene (9), eucalyptol (10), and $\alpha$-terpinene (11) were unambiguously confirmed by GC/MS analysis 
1 of the same extract. The strong point of this approach relies on the possibility to work with the

2 NMR tool directly on complex samples, which can be very useful to recover information about natural extract composition at a very early stage of the chemical profiling process. This can help

4 to design most reliable and appropriate experimental conditions for further metabolite 5 separation and to focus only on the relevant compounds depending on the target before 6 engaging in time-consuming multi-step purification procedures. The method can also be 7 considered as a rapid and highly complementary approach to mass spectrometry-based analytical tools such as MS infusion, LC/MS or GC/MS from which only molecular formula and fragmentation patterns are recovered. Additional work is currently taking place in our laboratory to further improve this dereplication process using not only $1 \mathrm{D}{ }^{13} \mathrm{C}$ NMR chemical 11 shifts data, but also 2D HSQC and HMBC NMR data from which ${ }^{1} \mathrm{H}_{-}{ }^{13} \mathrm{C}$ connectivity 12 information should greatly enhance the performance of the algorithm to identify more constituents in natural extracts.

\section{EXPERIMENTAL SECTION}

Chemicals, reagents, plant material. Methyl tert-butyl ether (MtBE), methanol, ethyl acetate, petroleum ether, chloroform, and acetonitrile $\left(\mathrm{CH}_{3} \mathrm{CN}\right)$ were purchased from Carlo Erba Reactifs SDS (Val de Reuil, France). Sodium hydroxide (NaOH), methane sulfonic acid (MSA) and triethylamine (TEA) were purchased from PROLABO (Paris, France). Deuterated methanol (methanol-d4) was obtained from Sigma-Aldrich (Saint-Quentin, France). Deionized 21 water was used to prepare all aqueous solutions.

23 Sample preparation. Peumus boldus dry leaves (500 g) purchased from Cailleau Herboristerie 24 (Chemillé-Melay, France) were ground into a fine powder and macerated for 24 hours in $10 \mathrm{~L}$ of petroleum ether for delipidation. Then, the marcs were alkalinized with $180 \mathrm{~mL}$ of $\mathrm{NH}_{3}$ 
1 (15\% in water) and macerated in ethyl acetate $(12 \mathrm{~L})$ for 24 hours. After leaching, three liquid-

2 liquid extractions were successively performed with $2 \mathrm{~L}$ of $\mathrm{H}_{2} \mathrm{SO}_{4}(0.5 \mathrm{~N})$ each time. The recovered aqueous phases were pooled and the $\mathrm{pH}$ was increased up to 9 with $\mathrm{NH}_{3}(15 \%$ in water). Three successive extractions were again performed with $2 \mathrm{~L}$ of chloroform each time. The organic phases were pooled and washed with $\mathrm{H}_{2} \mathrm{O}(2 \mathrm{~L})$. Chloroform was removed under vacuum to recover $3.5 \mathrm{~g}$ of a crude alkaloid extract.

8 Database search algorithm. The algorithm was implemented in the Python 2.7 programming

9 language. The open source cheminformatics package $\mathrm{RDKit}^{28}$ was used to draw the molecular structures and to read SDFiles. A literature survey was performed in order to obtain the names and the chemical structures of all the metabolites already described in the literature for the genus Peumus. A total of 58 molecular structures and their predicted ${ }^{13} \mathrm{C}$ NMR chemical shifts were 13 added to the spectral database (ACD/Labs, Ontario, Canada). Starting from the ${ }^{13} \mathrm{C}$ NMR peak list of the crude extract spectrum as input file, the principle of the algorithm is to perform a search over a part of the spectral database containing the metabolites of the genus under examination and to compare the ${ }^{13} \mathrm{C}$ NMR chemical shifts of the crude extract spectrum to the predicted chemical shifts of the database records. For each record, the algorithm constructs a list of NMR signals from the crude extract spectrum that matches signals of the record under examination (matching list). NMR signals are added to the matching list only if they satisfy two main conditions:

- ${ }^{13} \mathrm{C}$ NMR signals detected in the crude extract spectrum must have chemical shift values equal to the database record chemical shifts within a user defined tolerance of typically $2 \mathrm{ppm}(+/-1 \mathrm{ppm})$. This tolerance value is used in order to avoid false negatives due to variations between experimental and predicted chemical shifts. 
- ${ }^{13} \mathrm{C}$ NMR signals must have intensity values close to the mean intensity of all ${ }^{13} \mathrm{C}$ NMR signals to be considered as belonging to the same molecular structure. All signals with an intensity greater or lower than the mean intensity of the matching list $\left({ }^{13} \mathrm{C}\right.$ NMR peak list) by more than a user defined tolerance, typically 2 times the standard deviation around the mean intensity, are discarded.

Each match between database records and the crude extract spectrum is evaluated using a score

value calculated as followed: Score $=\frac{\sum \text { matching signals from the database molecule }}{\text { total number of the database molecule signals }}$

The score value is defined as the ratio between the numbers of chemical shifts of the crude extract that match a molecular structure of the database and the total number of carbon positions of this same record. Thus, the score values are comprised between 0 (no matching peaks between the crude extract spectrum and the database records) and 1 (a set of signals in the crude extract ${ }^{13} \mathrm{C}$ NMR spectrum matches all predicted chemical shifts of a database record).

The algorithm returns a list of metabolites sorted in the order of decreasing score. We assume that the records with the highest scores have the highest probability to be present in the sample, however this assumption is accepted only if all NMR signals of the proposed record are found in the mixture spectrum. Therefore a second search is performed for the top ranked records using a larger error tolerance on the chemical shifts $(+/-1.5 \mathrm{ppm})$ in order to check the presence of potentially missing peaks. The workflow of the algorithm is illustrated in Figure 1.

\section{Fractionation of the crude alkaloid extract by $\mathrm{pH}$-zone refining Centrifugal Partition}

Chromatography (CPC). The performance of the database search algorithm was evaluated by comparing the results to those obtained with a previously developed ${ }^{13} \mathrm{C} N M R$ based dereplication method involving a fractionation step. ${ }^{18}$ The crude alkaloid extract of Peumus boldus leaves was fractionated by $\mathrm{pH}-$ zone refining ${ }^{29} \mathrm{CPC}$ on a laboratory-scale FCPE300® (Kromaton Technology, Angers, France) equipped with a rotor made of 7 circular partition 
1 disks containing a total of 231 partition twin cells. The CPC column of $303.5 \mathrm{~mL}$ capacity was

2 connected to a KNAUER Preparative Pump 1800® V7115 (Berlin, Germany). Fractions of

$320 \mathrm{~mL}$ were collected by a Pharmacia Superfrac collector (Uppsala, Sweden). A biphasic

4 solvent system (3 L) was prepared by mixing into a separatory funnel $\mathrm{M} t \mathrm{BE}, \mathrm{CH}_{3} \mathrm{CN}$, and water

5 in the proportions 4/1/5 (v/v). After solvent system equilibration, the two liquid phases were

6 separated. Methane sulfonic acid (MSA) was used as retainer in the aqueous stationary phase

$7 \quad(4 \mathrm{mM}, \mathrm{pH} \approx 2)$ and triethylamine (TEA) was used as displacer in the mobile organic phase (5

$8 \mathrm{mM}, \mathrm{pH} \approx 10$ ). The column was filled at $200 \mathrm{rpm}$ with the acid aqueous stationary phase and

9 the rotation speed was then adjusted at $1200 \mathrm{rpm}$. The crude alkaloid extract of the leaves of Peumus boldus ( $2 \mathrm{~g})$ was dissolved in a $30 \mathrm{~mL}$ mixture of aqueous phase/TEA-free organic 11 phase in the proportions $1 / 1(\mathrm{v} / \mathrm{v})$. The sample was adjusted to $\mathrm{pH} 2$ with MSA before injection

to ensure alkaloid protonation. The sample solution was then loaded into the column through a $30 \mathrm{~mL}$ sample loop. The fractionation procedure was initiated by pumping progressively the alkaline organic mobile phase in the ascending mode from 0 to $20 \mathrm{~mL} / \mathrm{min}$ in $5 \mathrm{~min}$. The flow rate was then maintained at $20 \mathrm{~mL} / \mathrm{min}$ for 90 minutes. The collected fractions $(20 \mathrm{~mL}$ each) were checked by thin-layer chromatography (TLC) on Merck 60 F254 pre-coated silica gel plates and developed with chloroforme/methanol $(93: 7, \mathrm{v} / \mathrm{v})$. Detection was performed under UV light (254 and $366 \mathrm{~nm}$ ) and by spraying with the Dragendorff reagent. Fractions were then pooled on the basis of their TLC profile similarities, resulting in 11 pools noted from $\mathrm{P}_{\mathrm{I}}$ to $\mathrm{P}_{\mathrm{XI}}$.

NMR analyses and data processing. All NMR analyses were performed using identical acquisition and processing parameters. The crude alkaloid extract from Peumus boldus (15 mg) and the fractions $\mathrm{P}_{\mathrm{I}}$ to $\mathrm{P}_{\mathrm{XI}}$ obtained by CPC were dissolved in $600 \mu \mathrm{L}$ of DMSO-d6. NMR analyses were performed at $298 \mathrm{~K}$ on a Bruker Avance AVIII-600 spectrometer (Karlsruhe, Germany) equipped with a cryoprobe optimized for ${ }^{1} \mathrm{H}$ detection and with cooled ${ }^{1} \mathrm{H},{ }^{13} \mathrm{C}$ et ${ }^{2} \mathrm{D}$ 
1 coils and preamplifiers. ${ }^{13} \mathrm{C}$ NMR spectra were acquired at $150.91 \mathrm{MHz}$. A standard zgpg pulse

2 sequence was used with an acquisition time of $0.909 \mathrm{~s}$ and a relaxation delay of $3 \mathrm{~s}$. For each

3 sample, 1024 scans were co-added to obtain a satisfactory signal-to-noise $(\mathrm{S} / \mathrm{N})$ ratio. The

4 spectral width was $238.9070 \mathrm{ppm}$ and the receiver gain was set to the highest possible value. A

$5 \quad 1 \mathrm{~Hz}$ line broadening filter was applied to each FID prior to Fourier transformation. Spectra

6 were manually phased and baseline corrected using the TopSpin 3.2 software (Bruker) and

7 calibrated on the central resonance $(\delta 47.60 \mathrm{ppm})$ of methanol- $d 4$. The $\mathrm{S} / \mathrm{N}$ ratio of all spectra

8 was determined with the standard Bruker calculation method. Noise regions were selected from

9205 to $225 \mathrm{ppm}$ in all spectra and signal regions were selected differently for each spectrum as

10 the $10 \mathrm{ppm}$ spectral width centered on the most intense signal (methanol- $d 4$ apart). A minimum

11 intensity threshold of 0.05 was then used to automatically collect all positive ${ }^{13} \mathrm{C}$ NMR signals

12 while avoiding potential noise artifacts. The peak list obtained from the crude extract analysis

13 was exported as a text file and used as input file of the database search algorithm. The 11 peak

14 lists obtained from the CPC fraction series were also exported as text files and processed exactly

15 as previously described ${ }^{18}$.

16

17 GC/MS analysis. The crude alkaloid extract from Peumus boldus leaves was analyzed by

GC/MS. Chromatographic separation was carried out using a Trace 1300 gas chromatograph

(Thermo Scientific, Villebon sur Yvette, France) equipped with an AI 1310 injector. A TR5MS (Thermo Scientific) capillary column (30 m length, $0.25 \mathrm{~mm}$ internal diameter, and $210.25 \mu \mathrm{m}$ film thickness) was installed in the GC oven. The injection volume was $1 \mu \mathrm{L}$. Initially the temperature was set at $50{ }^{\circ} \mathrm{C}$ for 5 min followed by a $10{ }^{\circ} \mathrm{C} / \mathrm{min}$ ramp to $300{ }^{\circ} \mathrm{C}$. After $5 \mathrm{~min}$ at $300{ }^{\circ} \mathrm{C}$ the temperature was increased up to $310{ }^{\circ} \mathrm{C}$ with a ramp of $30^{\circ} \mathrm{C} / \mathrm{min}$ and stayed for 2 min. The detector was an ISQ Single Quadrupole mass spectrometer (Thermo Scientific). 
1 using the electron ionization (EI). The temperature of the transfer line and that of the ion source

2 were held at $250{ }^{\circ} \mathrm{C}$ and $230{ }^{\circ} \mathrm{C}$, respectively. The mass range $m / z$ 50-900 amu was scanned in

3 the full scan acquisition mode with a dwell time of $0.2 \mathrm{~s}$. Data were processed using the

4 Xcalibur software. The oil components were identified by comparing the MS spectra with

5 Libraries of NIST MS Search 2.0 program.

6

7 MS infusion analysis. An aliquot of the crude alkaloid extract from Peumus boldus leaves was

8 solubilized in methanol and directly infused in a quadrupole time-of-flight hybrid mass

9 spectrometer (QTOF micro ${ }^{\circledR}$, Waters, Manchester, UK) equipped with an electrospray source.

10 The mass range of the instrument was set at $\mathrm{m} / \mathrm{z} 100-1200$ and scan duration was set at $1 \mathrm{~s}$ in

11 the positive ion mode. The capillary voltage was $3000 \mathrm{~V}$, the cone voltage was $35 \mathrm{~V}$, and the

12 temperature was $80{ }^{\circ} \mathrm{C}$.

13

14 ASSOCIATED CONTENT

15 Supporting Information. Pseudocode of the database search algorithm

16

17 AUTHOR INFORMATION

18 Corresponding Author: *Jane Hubert

19 Jane.hubert@univ-reims.fr

21 NOTES

22 The authors declare no conflict of interest. 
1 The authors thank the Givaudan Company, the CNRS and the Ministry of Higher Education

2 and Research. The EU-programme FEDER for the PlAneT CPER project and the EU-Marie-

3 Curie Action for the IAPP/FP7-PEOPLE "Natprotec" project are also gratefully

4 acknowledged.

REFERENCES

8 (1) Gaudêncio, S. P.; Pereira, F. Nat. Prod. Rep. 2015, 32, 779-810.

9 (2) Hubert, J.; Nuzillard, J.-M.; Renault, J.-H. Phytochem. Rev. 2015, doi:10.1007/s11101015-9448-7.

11 (3) Wishart, D. S. Bioanalysis 2009, 1, 1579-1596.

12 (4) Carter, G. T. Nat. Prod. Rep. 2014, 31, 711-717.

13 (5) Nadia B, C.; Yu, K. Chromatogr. Online. 2013, pp 3-13.

14 (6) Garg, N.; Kapono, C. A.; Lim, Y. W.; Koyama, N.; Vermeij, M. J. A.; Conrad, D.;

Rohwer, F.; Dorrestein, P. C. Int. J. Mass Spectrom. 2015, 377, 719-727.

(7) Allard, P. M.; Péresse, T.; Bisson, J.; Gindro, K.; Marcourt, L.; Pham, V. C.; Roussi, F.; Wolfender, J. L. Anal. Chem. 2016, 88, 3317-3323.

(8) Armitage, E. G.; Barbas, C. J. Pharm. Biomed. Anal. 2014, 87, 1-11.

(9) Smolinska, A.; Blanchet, L.; Buydens, L. M. C.; Wijmenga, S. S. Anal. Chim. Acta 2012, 750, 82-97.

21 (10) Schripsema, J. Phytochem. Anal. 2010, 21, 14-21.

22 (11) Kim, H. K.; Choi, Y. H.; Verpoorte, R. Trends Biotechnol. 2011, 29 , 267-275.

23 (12) Krishnan, P.; Kruger, N. J.; Ratcliffe, R. G. J. Exp. Bot. 2005, 56, 255-265.

24 (13) Robinette, S. L.; Brüschweiler, R.; Schroeder, F. C.; Edison, A. S. Acc. Chem. Res. 2012, 45, 288-297. 
1 (14) Clendinen, C. S.; Lee-McMullen, B.; Williams, C.; Stupp, G. S.; Vandenborne, K.; Hahn, D. a; Walter, G. a; Edison, A. S. Anal. Chem. 2014, 86, 9242-9250.

(15) Laude, D. A.; Wilkins, C. L. Anal. Chem. 1986, 58, 2820-2824.

(16) Bighelli, A.; Tomi, F.; Casanova, J. Biomass and Bioenergy 1994, 6, 461-464.

(17) Ferreira, M. J. .; Costantin, M. B.; Sartorelli, P.; Rodrigues, G. V; Limberger, R.; Henriques, A. T.; Kato, M. J.; Emerenciano, V. P. Anal. Chim. Acta 2001, 447 , 125134.

(18) Hubert, J.; Nuzillard, J.-M.; Purson, S.; Hamzaoui, M.; Borie, N.; Reynaud, R.; Renault, J.-H. Anal. Chem. 2014, 86, 2955-2962.

(19) Oettl, S. K.; Hubert, J.; Nuzillard, J.-M.; Stuppner, H.; Renault, J.-H.; Rollinger, J. M. Anal. Chim. Acta 2014, 846, 60-67.

(20) Abedini, A.; Chollet, S.; Angelis, A.; Borie, N.; Nuzillard, J. M.; Skaltsounis, A. L.; Reynaud, R.; Gangloff, S. C.; Renault, J. H.; Hubert, J. J. Chromatogr. B 2016, 1029_ $1030,121-127$.

(21) Sientzoff, P.; Hubert, J.; Janin, C.; Voutquenne-Nazabadioko, L.; Renault, J.-H.; Nuzillard, J.-M.; Harakat, D.; Magid, A. Molecules 2015, 20, 14970-14984.

(22) Hubert, J.; Chollet, S.; Purson, S.; Reynaud, R.; Harakat, D.; Martinez, A.; Nuzillard, J.-M.; Renault, J.-H. J. Nat. Prod. 2015, 78, 1609-1617.

(23) Petigny, L.; Périno, S.; Minuti, M.; Visinoni, F.; Wajsman, J.; Chemat, F. Int. J. Mol. Sci. 2014, 15, 7183-7198.

(24) Bremser, W. Anal. Chim. Acta 1978, 2, 355-365.

(25) Buevich, A.V.; Elyashberg, E. J. Nat. Prod. 2016, 79, 3105-3116.

(26) Cimino, P.; Gomez-Paloma, L.; Duca, D.; Riccio, R.; Bifulco, G. Magn. Reson. Chem. 2004, 42, S26-S33.

(27) Bifulco, G.; Dambruoso, P.; Gomez-Paloma, L.; Riccio, R. Chem. Rev. 2007, 107, 
2 (28) RDKit http://www.rdkit.org/

(29) Okunji, C. O.; Iwu, M. M.; Ito, Y.; Smith, P. L. J. Liq. Chromatogr. Relat. Technol. 2005, 28, 775-783.

TABLES

Table 1. Proposals (structures and scores) of the database search algorithm applied to a crude extract of Peumus boldus leaves. 33 molecules with a score higher than "0.9", 28 molecules with a score of "1". Highlighted carbons correspond to ${ }^{13} \mathrm{C}$ chemical shifts that match peaks from the crude extract spectrum.

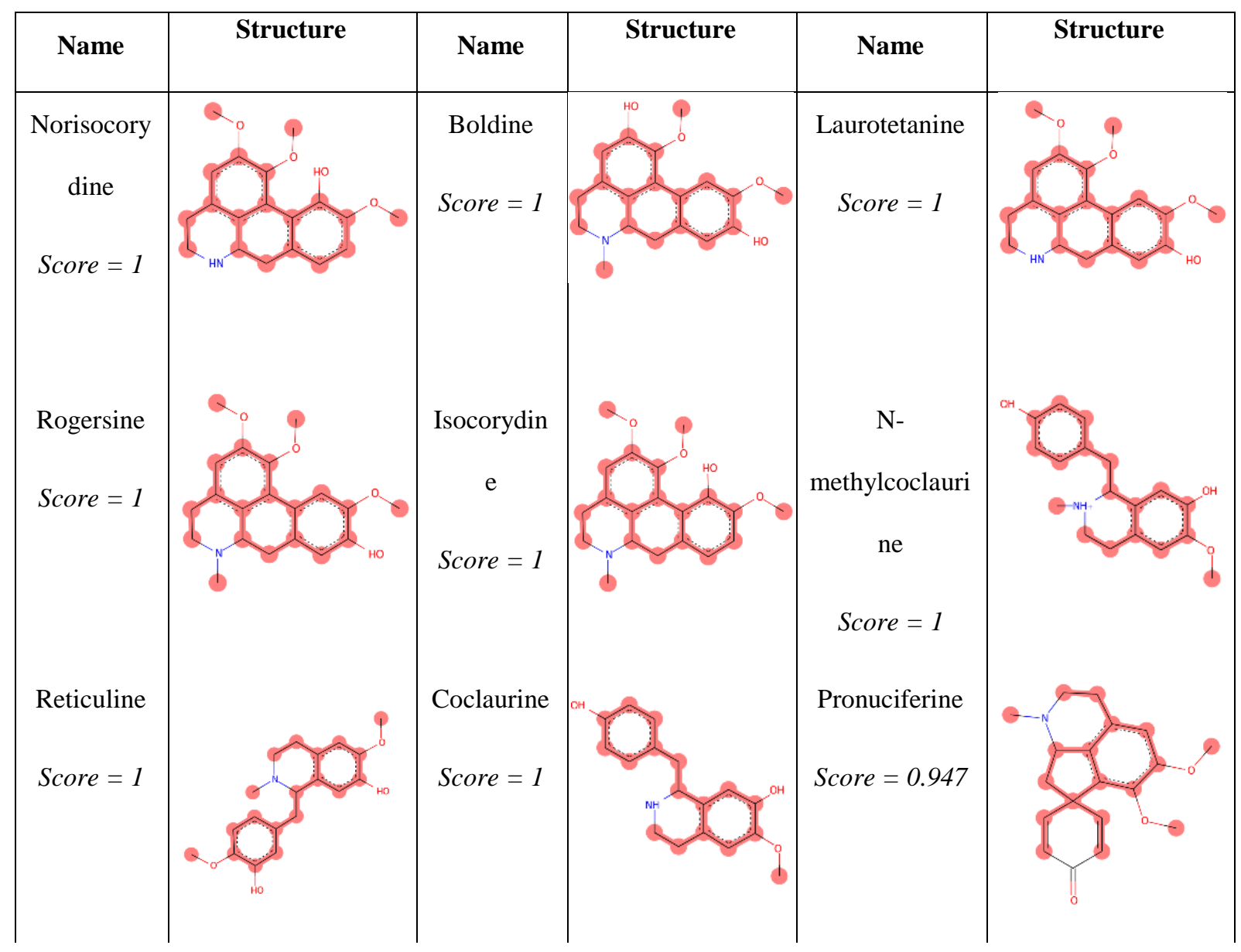




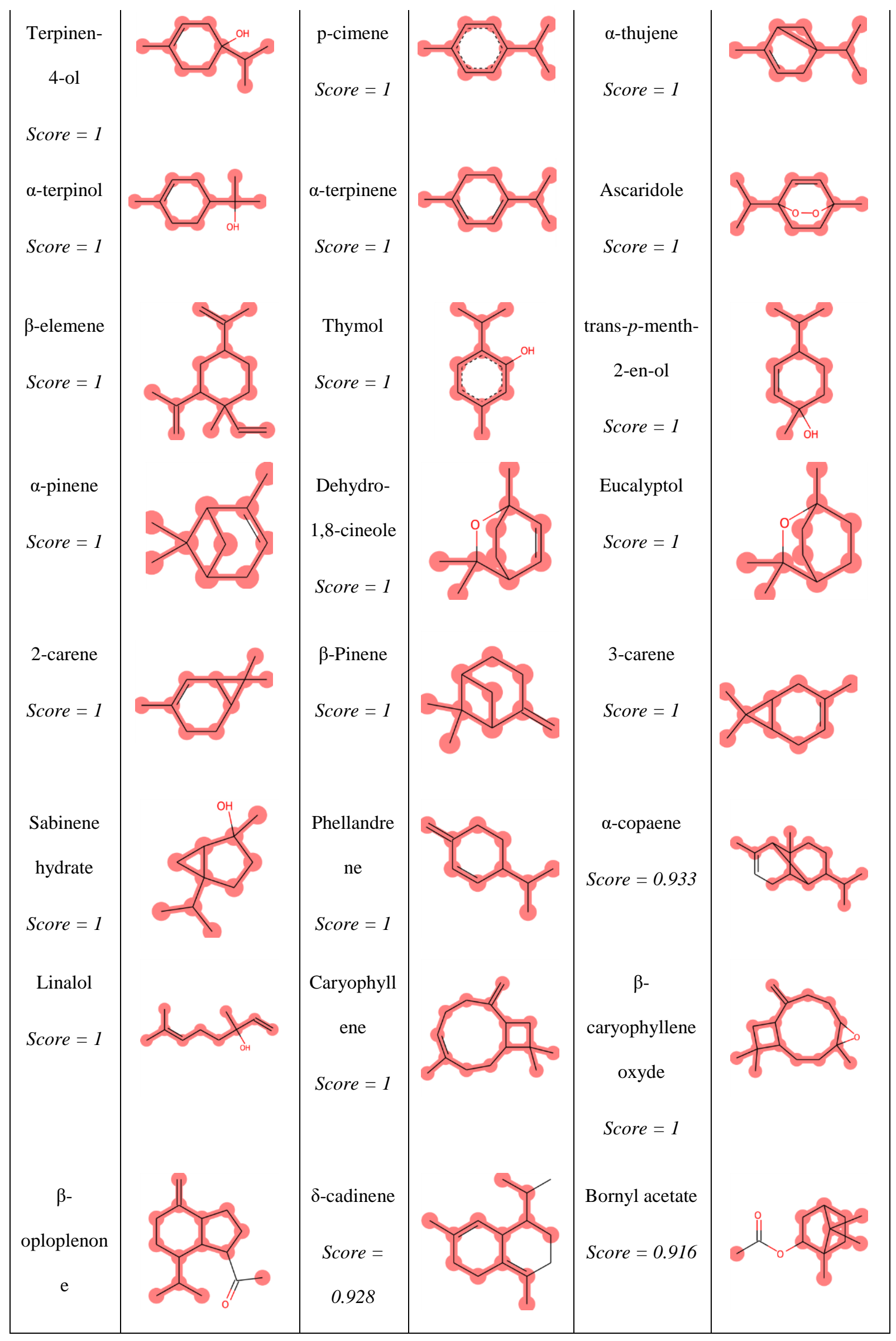




\begin{tabular}{|c|c|c|c|} 
Score $=$ \\
0.933
\end{tabular}

Table 2.

\begin{tabular}{|c|c|c|}
\hline $\begin{array}{l}\text { Retention } \\
\text { time (min) }\end{array}$ & $\begin{array}{c}\text { Identification } \\
\text { (Molecular formula) }\end{array}$ & MS spectra \\
\hline 3.2 & Residual DMSO & \\
\hline 5.7 & p-cymene $\left(\mathrm{C}_{10} \mathrm{H}_{14}\right)$ & 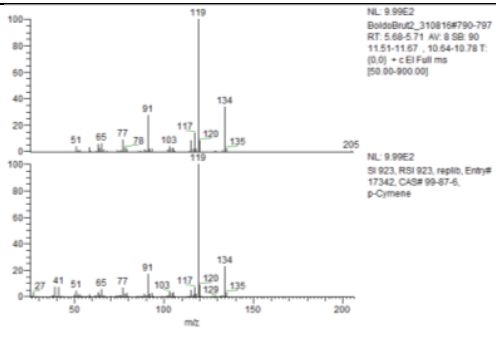 \\
\hline 5.82 & Eucalyptol $\left(\mathrm{C}_{10} \mathrm{H}_{18} \mathrm{O}\right)$ & 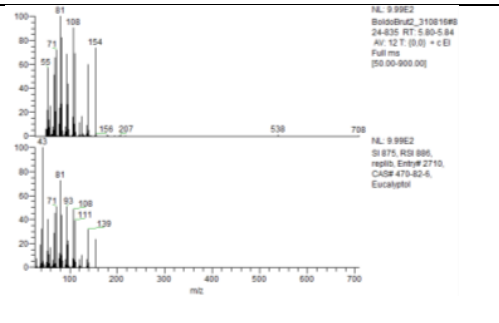 \\
\hline 8.1 & 3,7-octadiene-2,6-diol,2,6- & 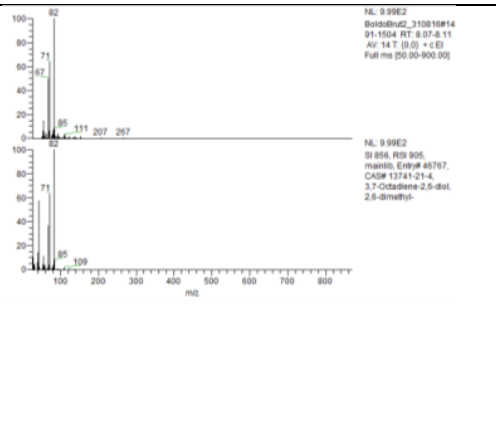 \\
\hline
\end{tabular}




\begin{tabular}{|c|c|c|}
\hline 9.0 & a-terpinene $\left(\mathrm{C}_{10} \mathrm{H}_{16}\right)$ & 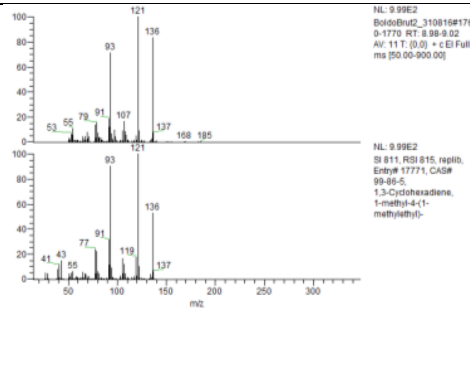 \\
\hline 9.3 & 1,7-octadiene-3,6-diol-2,6- & 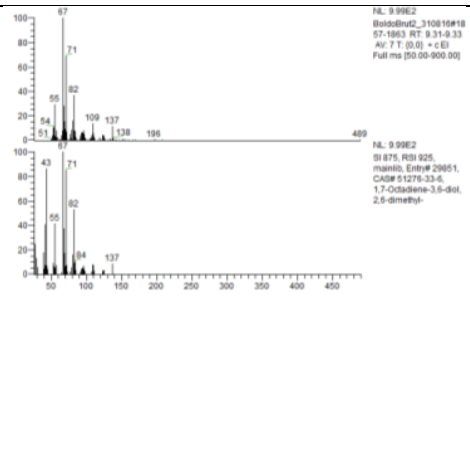 \\
\hline 9.4 & 1,4-dihydroxy- $p$-menth-2-ene & 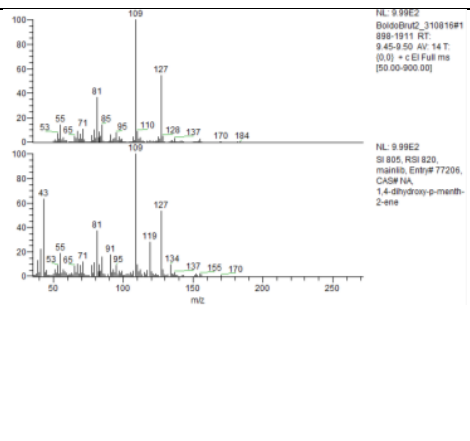 \\
\hline 9.7 & $\mathrm{C}_{10} \mathrm{H}_{18} \mathrm{O}_{2}$ & not identified \\
\hline 9.8 & $\mathrm{C}_{10} \mathrm{H}_{18} \mathrm{O}_{2}$ & not identified \\
\hline 10.1 & $\mathrm{C}_{10} \mathrm{H}_{18} \mathrm{O}_{2}$ & not identified \\
\hline 10.6 & $\mathrm{C}_{10} \mathrm{H}_{18} \mathrm{O}_{2}$ & not identified \\
\hline 11.0 & $\mathrm{C}_{10} \mathrm{H}_{16} \mathrm{O}$ & not identified \\
\hline 11.2 & $\mathrm{C}_{10} \mathrm{H}_{18} \mathrm{O}_{2}$ & not identified \\
\hline 11.3 & $\mathrm{C}_{10} \mathrm{H}_{16} \mathrm{O}_{2}$ & not identified \\
\hline 13.1 & $\mathrm{C}_{10} \mathrm{H}_{18} \mathrm{O}_{2}$ & not identified \\
\hline
\end{tabular}


Figure 1. ${ }^{13} \mathrm{C}$ NMR spectrum of the crude Peumus boldus extract with the two major alkaloids norisocorydine and rogersine annotated.

Figure 2. Workflow of the database search algorithm. (1) Chemical shift prediction for known metabolites for the studied genus and creation of the database. (2) ${ }^{13} \mathrm{C}$ NMR analysis of the crude extract and automatic peak picking. (3) Search algorithm: comparison of chemical shifts values of database records to those of the crude extract spectrum and prioritization of a list of putative molecules present within the crude extract. (**) Results confirmed by experimental analysis.

2 Figure 3. MS spectrum of the crude extract of Peumus boldus leaves (MS infusion, positive 3 ionization mode). A) $\mathrm{m} / z 286.1$ coclaurine $\mathrm{C}_{17} \mathrm{H}_{19} \mathrm{NO}_{3}$; B) $\mathrm{m} / z 300.1 \mathrm{~N}$-methylcoclaurine $4 \quad \mathrm{C}_{18} \mathrm{H}_{21} \mathrm{NO}_{3}$; C) $\mathrm{m} / \mathrm{z} 311.1$ not identified; D) $\mathrm{m} / \mathrm{z} 328.1$ norisocorydine OR boldine OR 5 laurotetanine $\mathrm{C}_{19} \mathrm{H}_{21} \mathrm{NO}_{4}$; E) $\mathrm{m} / z 330.1$ reticuline $\mathrm{C}_{19} \mathrm{H}_{23} \mathrm{NO}_{4}$; F) $\mathrm{m} / z, 342.1$ isocorydine OR 6 rogersine $\mathrm{C}_{20} \mathrm{H}_{23} \mathrm{NO}_{4}$.

$7 \quad$ Figure 4. ${ }^{13} \mathrm{C}$ NMR chemical shift clusters obtained by applying HCA on CPC fractions of 8 Peumus boldus.

9 Figure 5. Comparison between the ${ }^{13} \mathrm{C}$ NMR profiles of the crude extract of Peumus boldus

10 leaves and that of the $\mathrm{CPC}$ fractions (from $\mathrm{P}_{\mathrm{I}}$ to $\mathrm{P}_{\mathrm{XI}}$ ). $\mathrm{S} / \mathrm{N}$ : signal-to-noise ratio

11 Figure 6. GC/MS chromatogram obtained from the analysis of the crude extract of Peumus 12 boldus leaves. 
$1 \quad$ Figure 1.

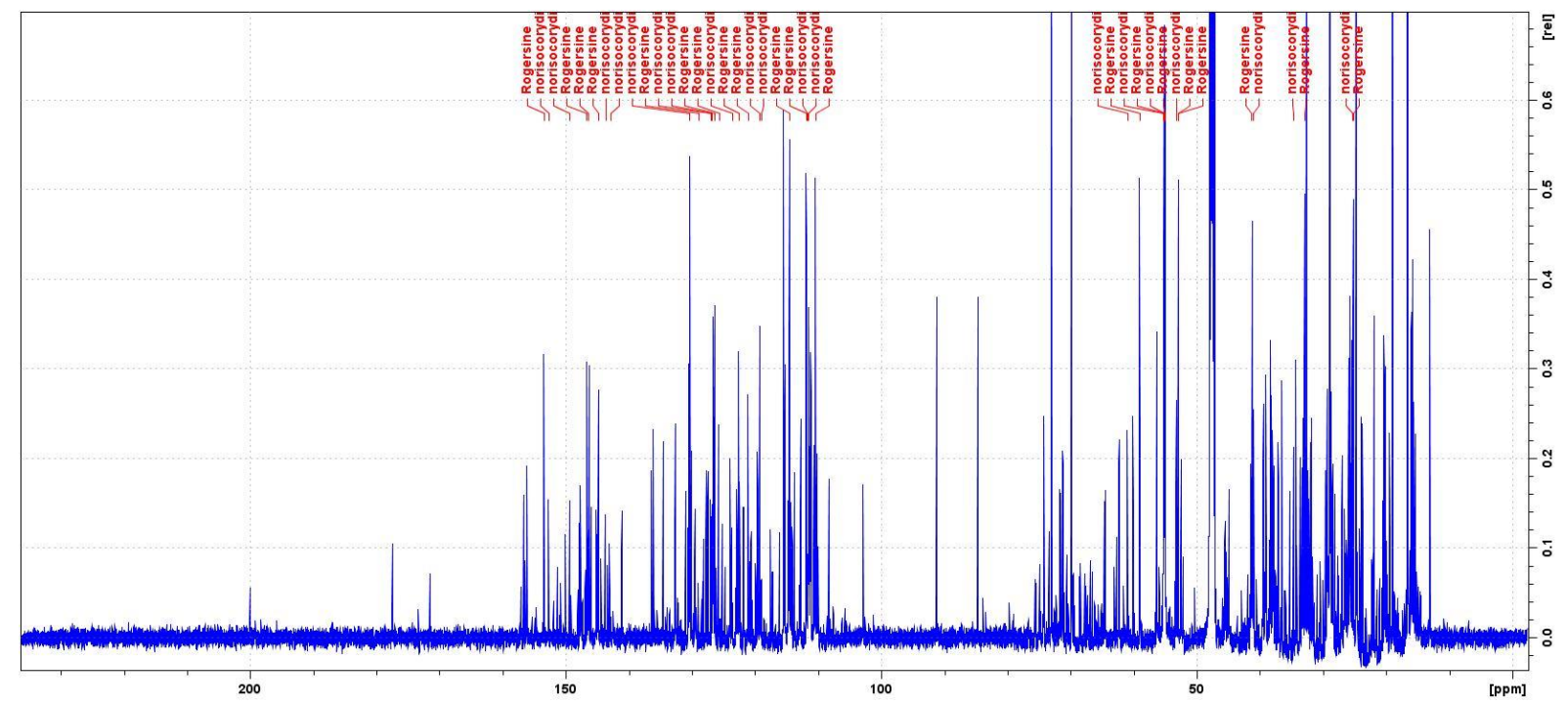

3 
Figure 2.

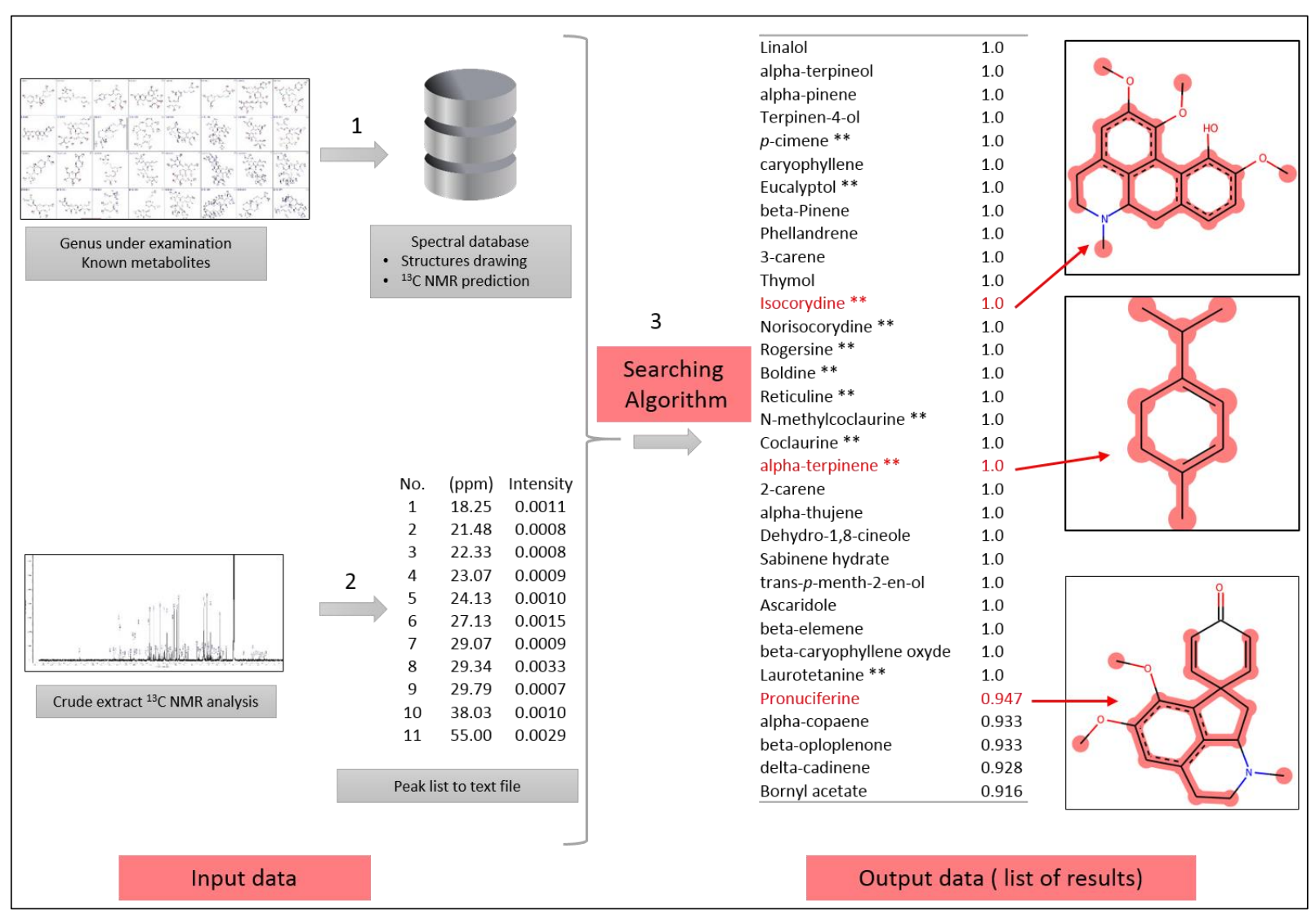

2

3

4 
Figure 3.

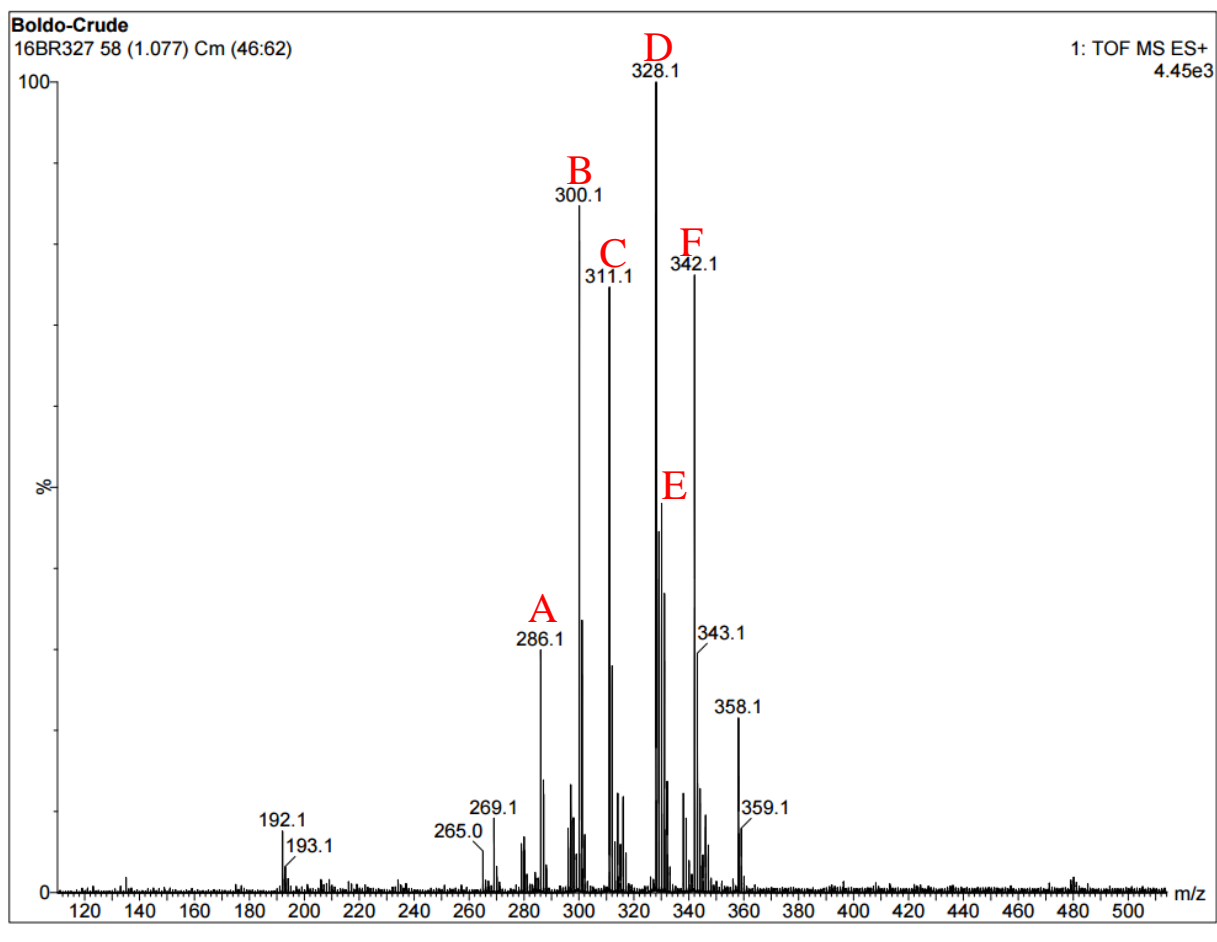

2 
Figure 4.

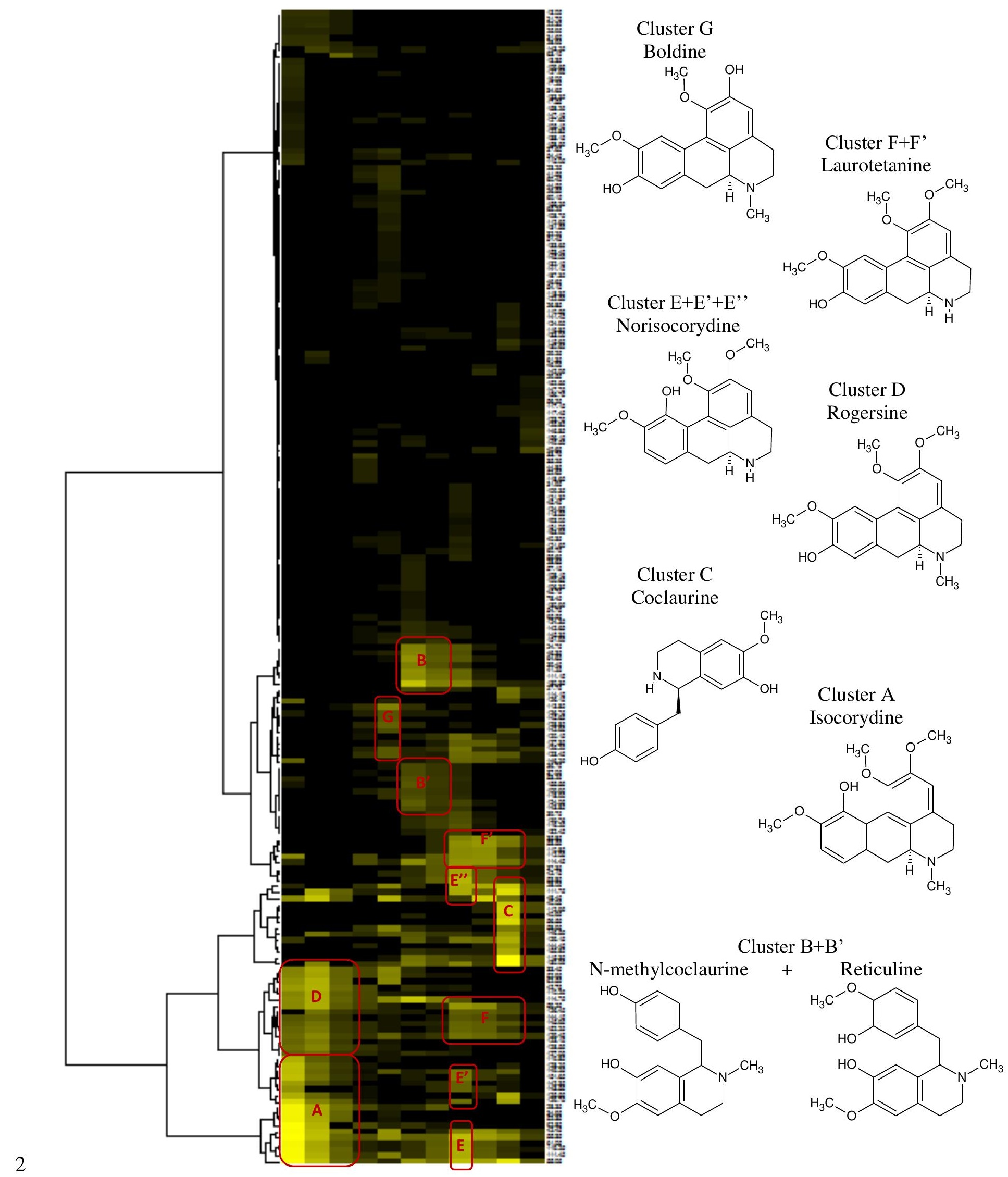


Figure 5.

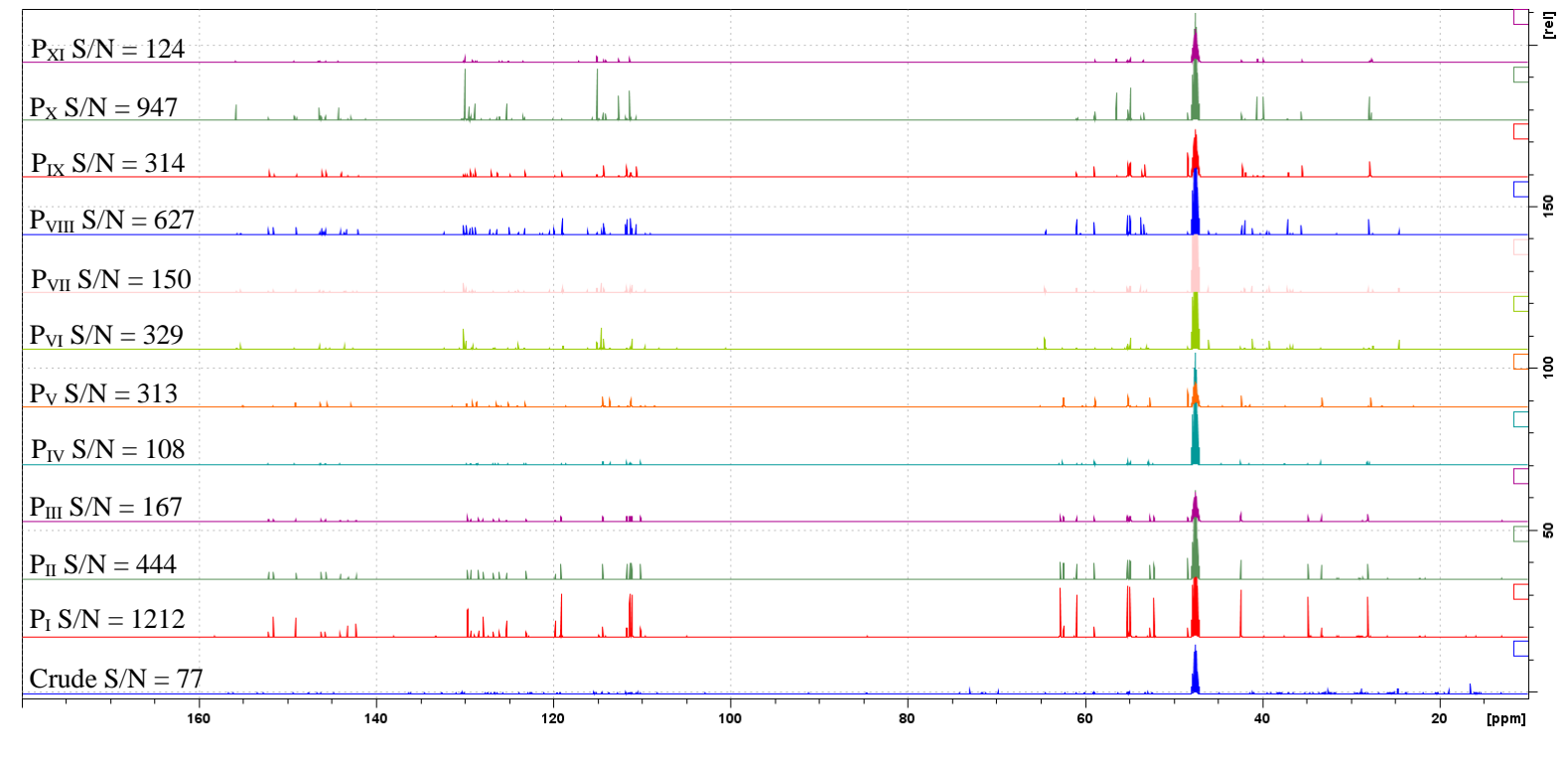

3 
Figure 6.

RT: $0.21-30.00$

$\mathrm{NL}$

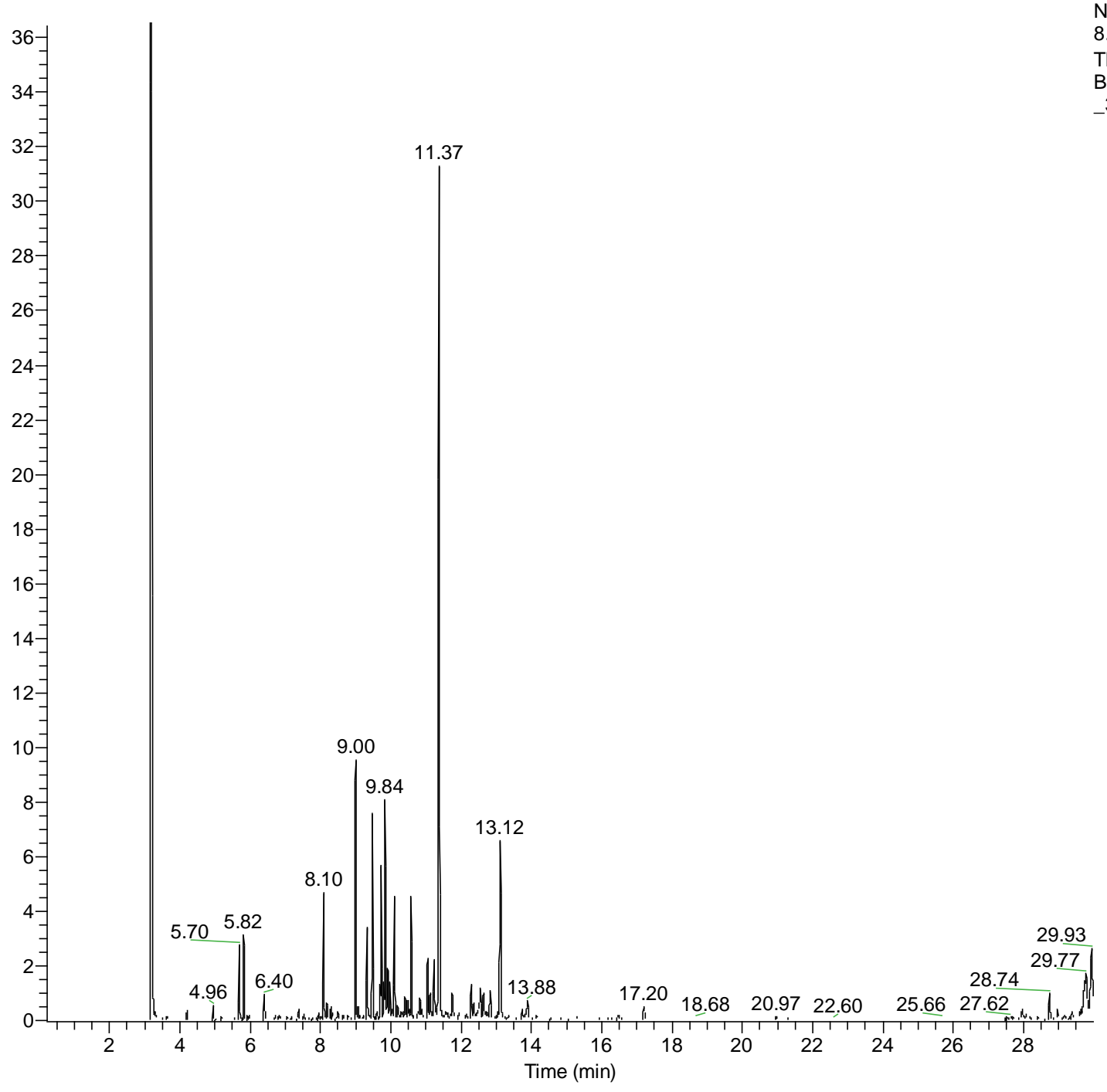

